\title{
Role of spin-orbit interaction in the production of spin-polarized photoelectrons using a dressing laser
}

\section{AUTHOR(S):}

Nakajima, T; Nikolopoulos, LAA

\section{CITATION:}

Nakajima, T ...[et al]. Role of spin-orbit interaction in the production of spin-polarized photoelectrons using a dressing laser. PHYSICAL REVIEW A 2003, 68(1): 013413.

ISSUE DATE:

2003-07

URL:

http://hdl.handle.net/2433/50416

RIGHT:

Copyright 2003 American Physical Society 
PHYSICAL REVIEW A 68, 013413 (2003)

\title{
Role of spin-orbit interaction in the production of spin-polarized photoelectrons using a dressing laser
}

\author{
Takashi Nakajima ${ }^{1, *}$ and L. A. A. Nikolopoulos ${ }^{2}$ \\ ${ }^{1}$ Institute of Advanced Energy, Kyoto University, Gokasho, Uji, Kyoto 611-0011, Japan \\ ${ }^{2}$ Institute of Electronic Structure and Laser, FORTH, P.O. Box 1527, Heraklion 711 10, Crete, Greece
}

(Received 3 April 2003; published 28 July 2003)

\begin{abstract}
We investigate the role of spin-orbit interaction in the production of spin-polarized photoelectrons under the influence of a dressing laser. Although the scheme considered here is essentially based on the laser-induced continuum structure, it turns out that special care has to be taken for the spin states in the continua. This is because the spin-orbit interaction in the continuum cannot be neglected for our specific case. After the derivation of the general formula, specific theoretical results are presented for $\mathrm{Rb}$ and $\mathrm{Cs}$ atoms. Enhancement about a factor of 2 in the degree of spin polarization has been predicted for both systems by the introduction of a dressing laser.
\end{abstract}

DOI: 10.1103/PhysRevA.68.013413

\section{INTRODUCTION}

Developing and searching for an efficient way to produce highly spin-polarized electrons has been one of the important issues in both basic and applied sciences, since the spin dependence of any physical phenomena provides additional information on the dynamics when one is to investigate various properties of gaseous as well as condensed matter. Recently a simple method has been proposed for the production of spinpolarized photoelectrons using multiphoton ionization of Xe [1]. The scheme is essentially based on quantum-mechanical interference which naturally has a dependence on laser frequency. For all single-, two-, and three-photon ionization schemes presented there, $>90 \%$ polarization has been predicted [1]. Furthermore, if the outgoing photoelectrons are spin polarized, electron spin of residual ions might be polarized as well. Two simple schemes have been proposed in Ref. [2] for the purpose of producing electron spin-polarized ions, and an experimental study is under progress [3].

It is now well known that a strong radiative coupling between an initially unoccupied excited state and a smooth continuum by the dressing laser can induce autoionizinglike resonance at a desired energy in the continuum. This is the so-called laser-induced continuum structure (LICS), and the theoretical prediction was reported some time ago [4-6], which was followed by experimental confirmations $[7,8]$. Much more clean experimental results together with a detailed theory have been reported in recent papers $[9,10]$.

The motivation of this study comes from an assumption that the LICS may be used to suppress (enhance) an undesired (desired) coherent ionization channel, which is an ionization into the spin-down or -up continuum, leading to the production of spin-polarized photoelectrons. Related to the study presented here, we should note that there are a few works in the literature $[11,12]$ which utilize the LICS for producing spin-polarized photoelectrons. The studies in there were within the framework of transition rate approximation.

In this paper, we investigate the role of spin-orbit interac-

\footnotetext{
*Email address: t-nakajima@iae.kyoto-u.ac.jp
}

PACS number(s): 32.80.Qk, 42.50.Hz, 32.80.Rm

tion in the continuum on the production of spin-polarized photoelectrons under the action of a dressing laser. We employ a set of time-dependent equations which is applicable up to the intensity of $\sim 10^{12} \mathrm{~W} / \mathrm{cm}^{2}$. Various ionization channels and ac Stark shifts are taken into account. In particular, special attention is paid to the description of continua in terms of spin states. Note that the main objective of this paper is to understand the underlying mechanism of producing spin-polarized photoelectrons under the action of a dressing laser, rather than to propose and assess alternative means for the production of spin-polarized photoelectrons.

The level scheme we consider is shown in Fig. 1. The ground state of alkali-metal atoms, ${ }^{2} S_{1 / 2}\left(m_{j}= \pm 1 / 2\right)$ denoted as $|0\rangle$ with energy $E_{0}$, is coupled to the continuum by a right-circularly polarized probe laser with frequency $\omega_{p}$. An initially unoccupied excited state, ${ }^{2} S_{1 / 2}\left(m_{j}= \pm 1 / 2\right)$ denoted as $|1\rangle$ with energy $E_{1}$, is also coupled to the continuum by a linearly polarized dressing laser with frequency $\omega_{d}$. Note that the two lasers are assumed to be in a crossbeam geometry at right angle, so that the quantization axis, which is conventionally taken along the propagation direction for circular light and the polarization direction for linear light, can be defined to be the same for both lasers. The frequencies of the lasers are chosen in such a way that a two-photon near-resonance is realized, i.e., $E_{0}+\hbar \omega_{p} \simeq E_{1}$ $+\hbar \omega_{d}$. Due to the dipole selection rule, only the $p$ continuum can be reached from the ground and also from the excited ${ }^{2} S_{1 / 2}$ states. At first glance, it appears that the underlying physics can be well described by the scheme depicted in Fig. 1(a). The naive scenario we had in mind before the detailed study is as follows. Due to the LICS via the "apparently" common $p$ continuum denoted as $m_{j}=1 / 2$ in Fig. 1(a), the ionization channel from the ground ${ }^{2} S_{1 / 2}\left(m_{j}=-1 / 2\right)$ state can be completely turned off at a certain detuning $\delta$. What is left is the ionization channel from the ground ${ }^{2} S_{1 / 2}\left(m_{j}=1 / 2\right)$ state, photoelectrons from which are $100 \%$ spin up. After a more careful investigation, however, we came across a problem: Recall that the valence electron in ${ }^{2} S_{1 / 2}\left(m_{j}=-1 / 2\right)$ is necessarily spin down. As long as the spin-orbit interaction is not taken into account, the spin state will not change, irrespective of the polarization of the lasers 
T. NAKAJIMA AND L. A. A. NIKOLOPOULOS

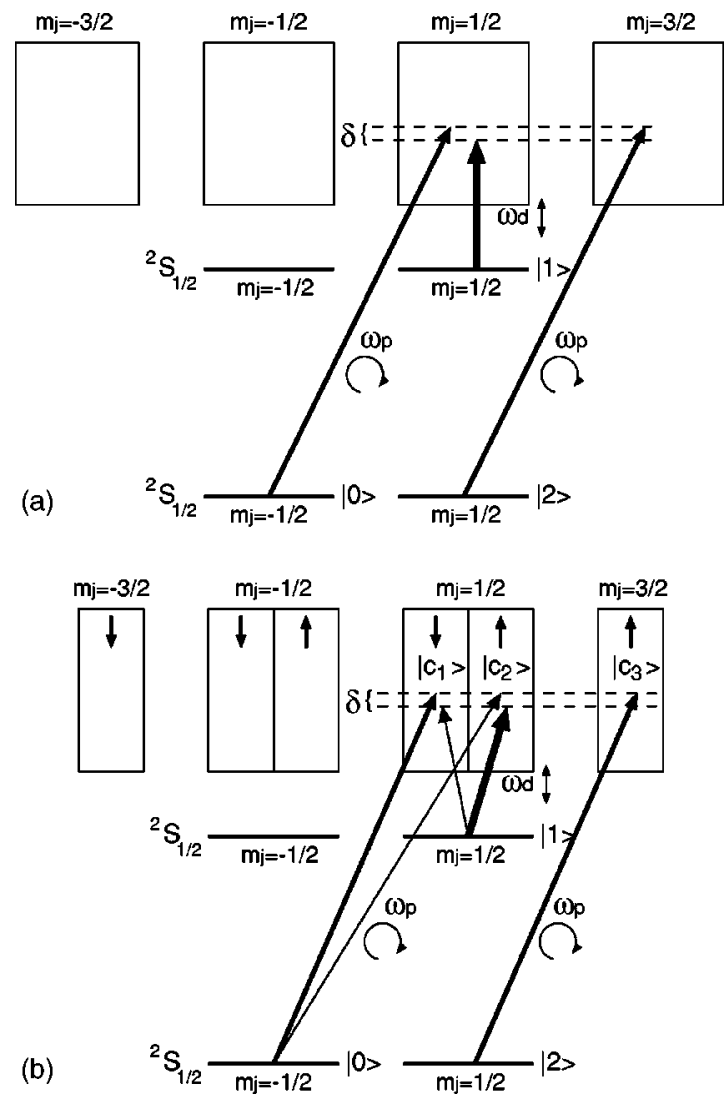

FIG. 1. Level scheme for the case in which the probe laser is right-circularly polarized, while the dressing laser is linearly polarized. (a) Naive (and misleading) picture. (b) Correct picture for which detailed results are presented in this paper. Line thickness in (b) indicates coupling strength.

used for excitation/ionization. This simple argument lead us into a dilemma of how the transitions by the probe and dressing lasers in Fig. 1(a) can bring atoms to the common $p$ continuum with $m_{j}=1 / 2$. The answer to this dilemma is that the picture described in Fig. 1(a) is misleading. The correct physical picture is illustrated in Fig. 1(b). Briefly, the transitions from the ground ${ }^{2} S_{1 / 2}\left(m_{j}=-1 / 2\right)$ state and the excited ${ }^{2} S_{1 / 2}\left(m_{j}=1 / 2\right)$ state by the right-circularly polarized probe and linearly polarized dressing lasers do not go to the same $p$ continuum with $m_{j}=1 / 2$. Rather, transitions bring atoms into the different spin states belonging to the same $p$ continuum with $m_{j}=1 / 2$, as depicted in Fig. 1(b). In the limit of no spin-orbit interaction, the transitions drawn by thin lines in Fig. 1(b) vanish, and hence there is no LICS at all. In other words, in order for the system to exhibit a LICS, it is essential that there is sufficient spin-orbit interaction in the continuum.

For better understanding, it is instructive to consider the case in which both lasers are linearly polarized, as often employed in LICS experiments [7-10]. Note that photoelectrons are not spin polarized in this case, since there is no net angular momentum transferred from light to atoms. A naive (and again misleading) picture and a correct picture are depicted in Figs. 2(a) and 2(b), respectively. Even if there is no spin-orbit interaction in the continuum, i.e., no radiative cou-
PHYSICAL REVIEW A 68, 013413 (2003)

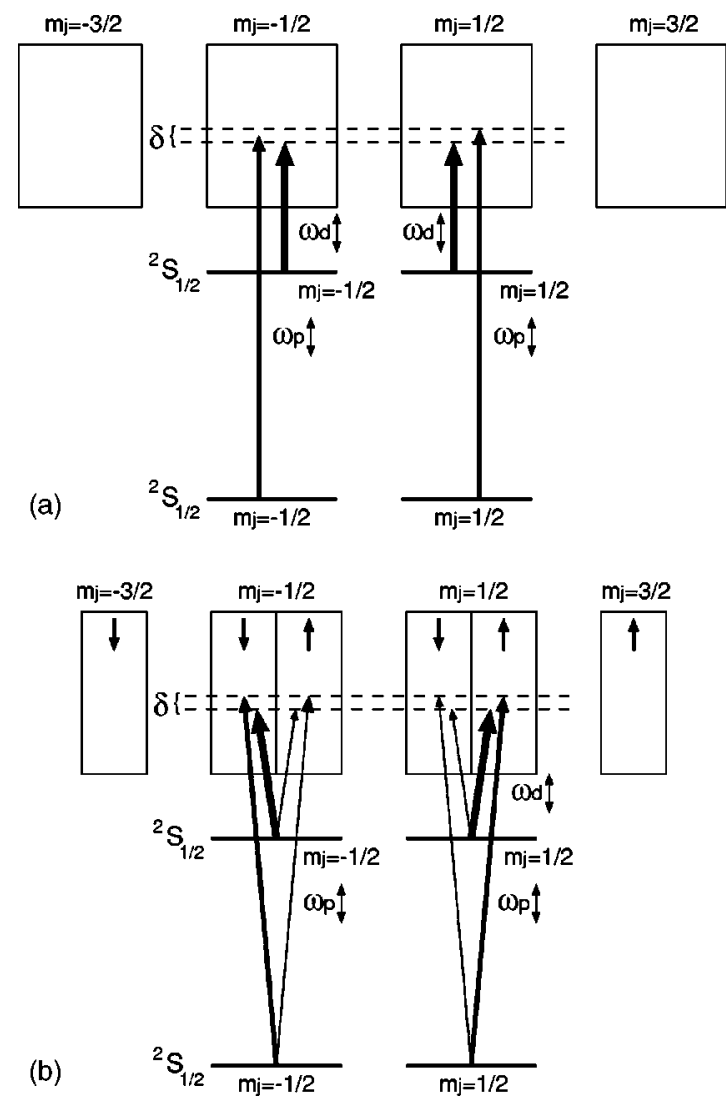

FIG. 2. Level scheme for the case in which both lasers are linearly polarized. (a) Naive (and misleading) picture. (b) Correct picture. Line thickness in (b) indicates coupling strength.

plings depicted by thin lines in Fig. 2(b), the LICS can be still observed. Although the spin-orbit interaction brings a bound electron to the spin-flipped continuum with a small probability, its contribution to the LICS is much smaller in this case, and does not change the basic feature of the LICS spectrum. Therefore, neglecting the spin-orbit interaction is considered to be a rather good approximation as long as both lasers are linearly polarized. A similar argument holds for the case in which both lasers are circularly polarized.

\section{THEORY}

In order to describe all the dynamics involved in Fig. 1(b) in a time-dependent manner, we employ a resolvent operator formalism. In the following section, we carry out specific calculations for $\mathrm{Rb}\left[5 s_{1 / 2}\left(m_{j}=-1 / 2\right)\right.$ for $|0\rangle$ and $7 s_{1 / 2}\left(m_{j}\right.$ $=1 / 2)$ for $|1\rangle]$ and $\mathrm{Cs}\left[6 s_{1 / 2}\left(m_{j}=-1 / 2\right)\right.$ for $|0\rangle$ and $8 s_{1 / 2}\left(m_{j}=1 / 2\right)$ for $\left.|1\rangle\right]$. Before going into the detail of the model, we note that the hyperfine structure of the system has been neglected throughout this work, which will be justified in Sec. III B.

Now, we start with the resolvent equation $[13,14]$

$$
\left(z-H_{0}-D^{(p)}-D^{(d)}\right) G=1,
$$

where $H_{0}$ is a field-free Hamiltonian, and $D^{(p)}$ and $D^{(d)}$ are the dipole operators for the probe and dressing fields, respec- 
tively. Following the standard procedure [13,14], a set of resolvent equations is obtained as follows:

$$
\begin{aligned}
& {\left[z-E_{0}-\hbar \omega_{p}+\hbar \omega_{d}-\left(S_{0}^{(p)}+S_{0}^{(d)}\right)+i \frac{\Gamma_{0}^{(p)}}{2}\right]} \\
& \quad \times G_{0}-\Omega\left(1-\frac{i}{q}\right) G_{1}=1 \\
& -\Omega\left(1-\frac{i}{q}\right) G_{0}+\left[z-E_{1}-\left(S_{1}^{(p)}+S_{1}^{(d)}\right)+\frac{i}{2}\left(\Gamma_{1}^{(p)}+\Gamma_{1}^{(d)}\right)\right] \\
& \times G_{1}=0
\end{aligned}
$$

where all the continua $\left|c_{k}\right\rangle(k=1,2,3,4)$ have been eliminated. The continuum state $\left|c_{4}\right\rangle$, not shown in Fig. 1(b), is a continuum belonging to the same parity and the spin state as $\left|c_{3}\right\rangle$, but at a different energy, since this is the continuum reached by the single-photon absorption of the probe laser from state $|1\rangle . \Gamma_{j}^{(\alpha)}$,s are the total ionization widths of state $|j\rangle(j=0$ or 1$)$ by the probe $(\alpha=p)$ or dressing laser $(\alpha$ $=d$ ) into all the possible continua. The partial ionization widths such as $\Gamma_{0 c_{k}}^{(p)}$ into each continuum $\left|c_{k}\right\rangle(k=1,2,3,4)$ are implicit in $\Gamma_{j}^{(\alpha)}$, and they are connected through the relation

$$
\Gamma_{j}^{(\alpha)}=\sum_{k=c_{1} \text { to } c_{4}} \Gamma_{j k}^{(\alpha)}(j=0,1,2) .
$$

$\Omega$ is a two-photon Rabi frequency between $|0\rangle$ and $|1\rangle$, and $q$ is an asymmetry parameter. The two-photon Rabi frequency $\Omega$ is a sum of the two-photon Rabi frequencies coupled via spin-down/spin-up continuum, $\left|c_{1}\right\rangle$ and $\left|c_{2}\right\rangle$, i.e.,

$$
\Omega\left(1-\frac{i}{q}\right)=\sum_{\beta=c_{1}, c_{2}} \Omega_{\beta}\left(1-\frac{i}{q_{\beta}}\right) .
$$

$S_{j}^{(\alpha)}$,s are the ac Stark shifts of state $|j\rangle$ by laser $p$ or $d$. Note that the spontaneous decay of $|1\rangle$ has been neglected in this work, since the natural lifetimes of $\mathrm{Rb} 7 s_{1 / 2}$ and $\mathrm{Cs} 8 s_{1 / 2}$ are $88 \mathrm{~ns}$ and $104 \mathrm{~ns}$, respectively, which are much longer than the laser pulse duration ( $1 \mathrm{~ns}-10 \mathrm{~ns})$ we assume in this paper.

For the time-varying laser intensities, we have to convert the resolvent equations into the amplitude equations. They read

$$
\begin{gathered}
\dot{u}_{0}=-\frac{1}{2}\left(\Gamma_{0 c_{1}}^{(p)}+\Gamma_{0 c_{2}}^{(p)}\right) u_{0}-i \Omega\left(1-\frac{i}{q}\right) u_{1}, \\
\dot{u}_{1}=\left[i \delta-\frac{1}{2}\left(\Gamma_{1 c_{4}}^{(p)}+\Gamma_{1 c_{1}}^{(d)}+\Gamma_{1 c_{2}}^{(d)}\right)\right] u_{1}-i \Omega\left(1-\frac{i}{q}\right) u_{0}, \\
\dot{u}_{2}=-\frac{1}{2} \Gamma_{2 c_{3}}^{(p)} u_{2},
\end{gathered}
$$

where $u_{j}$ 's $(j=0,1,2)$ are the probability amplitudes of states $|j\rangle . \quad \delta$ is the two-photon detuning defined by $\delta=\left(E_{0}\right.$ $\left.+\hbar \omega_{p}\right)-\left(E_{1}+\hbar \omega_{d}\right) . D_{j k}^{(\alpha)}$, s are the bound-free matrix ele- ments by laser $\alpha$ from the bound state $|j\rangle$ to the continuum $|k\rangle$, which are connected to the ionization widths by the relation $\Gamma_{j k}^{(\alpha)}=2 \pi\left|D_{j k}^{(\alpha)}\right|^{2}$.

Photoelectron yield into each continuum can be computed using the relations

$$
\begin{aligned}
& R_{c_{1}}(t)= \int_{-\infty}^{t} d t^{\prime}\left\{\Gamma_{0 c_{1}}^{(p)}\left|u_{0}\right|^{2}+\Gamma_{1 c_{1}}^{(d)}\left|u_{1}\right|^{2}\right. \\
&\left.+2 \operatorname{Im}\left[\Omega_{c_{1}}\left(1+\frac{i}{q_{c_{1}}}\right)\left(u_{0} u_{1}^{*}+u_{1} u_{0}^{*}\right)\right]\right\}, \\
& R_{c_{2}}(t)= \int_{-\infty}^{t} d t^{\prime}\left\{\Gamma_{0 c_{2}}^{(p)}\left|u_{0}\right|^{2}+\Gamma_{1 c_{2}}^{(d)}\left|u_{1}\right|^{2}\right. \\
&\left.+2 \operatorname{Im}\left[\Omega_{c_{2}}\left(1+\frac{i}{q_{c_{2}}}\right)\left(u_{0} u_{1}^{*}+u_{1} u_{0}^{*}\right)\right]\right\}, \\
& R_{c_{3}}(t)=\int_{-\infty}^{t} d t^{\prime} \Gamma_{1 c_{3}}^{(p)}\left|u_{1}\right|^{2}, \\
& R_{c_{4}}(t)=\int_{-\infty}^{t} d t^{\prime} \Gamma_{2 c_{4}}^{(p)}\left|u_{2}\right|^{2} .
\end{aligned}
$$

\section{NUMERICAL RESULTS}

\section{A. Calculation of matrix elements}

First, we calculate necessary matrix elements for $\mathrm{Rb}$ and Cs. In particular, we have chosen $\operatorname{Rb~} 5 s_{1 / 2}\left(m_{j}=-1 / 2\right)$, $7 s_{1 / 2}\left(m_{j}=1 / 2\right), \quad$ and $\quad 5 s_{1 / 2}\left(m_{j}=1 / 2\right), \quad\left[\operatorname{Cs} 6 s_{1 / 2}\left(m_{j}=\right.\right.$ $-1 / 2), 8 s_{1 / 2}\left(m_{j}=1 / 2\right)$, and $\left.6 s_{1 / 2}\left(m_{j}=1 / 2\right)\right]$ for $|0\rangle,|1\rangle$, and $|2\rangle$, respectively. The wavelength of the dressing laser is chosen to be $1.06 \mu \mathrm{m}$, which corresponds to an Nd:YAG (yttrium aluminum garnet) laser. As for the probe laser, the wavelength must be chosen such that nearly two-photon resonance is realized, i.e., $\delta \sim 0$.

It should be clear by now that the matrix elements we need must be $j$ dependent, since spin-orbit interaction plays an essential role in our specific case (see Appendixes A-C). The most rigorous treatment of spin-orbit interaction is to solve the Dirac equation. An alternative way is to include a spin-orbit term in the nonrelativistic Hamiltonian. In this work, however, we employ a simpler method. We have employed pseudopotentials to calculate wave functions of the valence electron of $\mathrm{Rb}$ and $\mathrm{Cs}$. It is essential that our pseudopotentials have $j$ as well as $l$ dependence.

Usually, the radial component of nonrelativistic oneelectron Schrödinger equation in a central field can be described using an $l$-dependent pseudopotential $V_{l}(r)$ and a core polarization. The potential $V_{l}(r)$ describes an interaction of valence electrons with the core. Once $V_{l}(r)$ and the core polarization are given, a series of radial functions and corresponding eigenenergies can be obtained for each $l$ by solving the one-electron Schrödinger equation. Of course, if the potential $V_{l}(r)$ has a different form, different radial functions and eigenenergies are obtained. In this way one can fit the form of pseudopotentials so that the calculated energies agree with the experimental ones. Furthermore, by introduc- 
TABLE I. Semiempirical parameters in Eq. (14) for Rb for the pseudopotential.

\begin{tabular}{lccccc}
\hline \hline & $a_{l j}$ & $b_{l j}$ & $c_{l j}$ & $d_{l j}$ & $r_{c_{l j}}$ \\
\hline Rb $s_{1 / 2}$ & -26.1762 & 1.6970 & -3.3004 & 1.8627 & 3.4429 \\
$p_{1 / 2}$ & -26.1612 & 1.2324 & -3.0172 & 1.9651 & 1.5952 \\
$p_{3 / 2}$ & -26.2561 & 1.2159 & -3.0569 & 2.0728 & 1.7321 \\
\hline \hline
\end{tabular}

ing $j$ dependence of eigenenergies during fittings, the radial functions become $j$ dependent as well. In other words, one can phenomenologically introduce spin-orbit interactions through $j$-dependent pseudopotentials. That is,

$$
\left[-\frac{1}{2} \frac{d^{2}}{d r^{2}}+V_{l j}(r)\right] P_{n l j}(r)=\epsilon_{n l j} P_{n l j}(r),
$$

where $P_{n l j}(r)$ is a $j$-dependent one-electron radial function and $\epsilon_{n l j}$ is a $j$-dependent eigenenergy. The pseudopotential $V_{l j}(r)$ we employ has a form of

$$
\begin{aligned}
V_{l j}(r)= & -\frac{1}{r}+\frac{l(l+1)}{2 r^{2}}-\frac{a_{l j} \exp \left(-b_{l j} r\right)}{r}-c_{l j} \exp \left(-d_{l j} r\right) \\
& -\frac{\alpha_{d}}{r^{4}}\left\{1-\exp \left[-\left(\frac{r}{r_{c_{l j}}}\right)^{6}\right]\right\}
\end{aligned}
$$

The third and fourth terms in Eq. (14) correspond to the short-range interaction, representing the interaction of the valence electron with the core, with spin-orbit interaction phenomenologically taken into account. The last term in Eq. (14) describes a dipole polarization with a correct asymptotic form. $r_{c_{l j}}$ is a cutoff parameter and $\alpha_{d}$ is a dipole polarizability of the core $\mathrm{Rb}^{+}$or $\mathrm{Cs}^{+}$. The values of the dipole polarizabilities $\alpha_{d}$ 's are taken from the literature [15], which are 9.076 and 15.81 for $\mathrm{Rb}^{+}$and $\mathrm{Cs}^{+}$, respectively. Fitting of parameters $a_{l j}, b_{l j}, c_{l j}, d_{l j}$, and $r_{c_{l j}}$ have been carried out for the lowest four states in each $s_{1 / 2}, p_{1 / 2}$, and $p_{3 / 2}$ series. In order for the fitted parameters to be consistent as much as possible, fittings for $p_{1 / 2}$ and $p_{3 / 2}$ are first performed for the statistically averaged $p$ series. Then, starting from those parameters, fittings are further performed for each $p_{1 / 2}$ and $p_{3 / 2}$ series. Thus, fitted parameters are listed in Tables I and II for $\mathrm{Rb}$ and $\mathrm{Cs}$. After the parameter fittings for the pseudopotentials, the calculated energies agree with the experimental ones for the lowest six states of each $s_{1 / 2}, p_{1 / 2}$, and $p_{3 / 2}$ series within the accuracy of $<1 \mathrm{~cm}^{-1}$ for $\mathrm{Rb}$ and $<2.5 \mathrm{~cm}^{-1}$ for Cs.

TABLE II. Semiempirical parameters in Eq. (14) for Cs for the pseudopotential.

\begin{tabular}{lrcrcc}
\hline \hline & \multicolumn{1}{c}{$a_{l j}$} & $b_{l j}$ & \multicolumn{1}{c}{$c_{l j}$} & $d_{l j}$ & $r_{c_{l j}}$ \\
\hline Cs $s_{1 / 2}$ & 3.0822 & 1.9514 & -27.4598 & 1.6987 & 3.4147 \\
$p_{1 / 2}$ & -2.0043 & 0.9579 & -7.4053 & 1.2161 & 4.2557 \\
$p_{3 / 2}$ & -4.8884 & 0.9483 & -9.5531 & 1.2497 & 3.5271 \\
\hline \hline
\end{tabular}

TABLE III. Atomic parameters for Rb $5 s-7 s$ system. $\Omega$ in $\mathrm{rad} / \mathrm{s}$, $\Gamma$ in $\mathrm{s}^{-1}, S$ in $\mathrm{rad} / \mathrm{s}$, and $I_{d}$ in $\mathrm{W} / \mathrm{cm}^{2}$.

\begin{tabular}{lccc}
\hline \hline$\Omega_{c_{1}}$ & $-1.37 \sqrt{I_{p} I_{d}}$ & $q_{c_{1}}$ & -17.5 \\
$\Omega_{c_{2}}$ & $2.43 \sqrt{I_{p} I_{d}}$ & $q_{c_{2}}$ & 24.1 \\
\hline$\Gamma_{0 c_{1}}^{(p)}$ & $0.522 I_{p}$ & $\Gamma_{1 c_{1}}^{(d)}$ & $0.0469 I_{d}$ \\
$\Gamma_{0 c_{2}}^{(p)}$ & $0.00796 I_{p}$ & $\Gamma_{1 c_{2}}^{(d)}$ & $5.069 I_{d}$ \\
$\Gamma_{2 c_{4}}^{(p)}$ & $0.72 I_{p}$ & $\Gamma_{1 c_{3}}^{(p)}$ & $5.2 \times 10^{-4} I_{p}$ \\
$S_{0}^{(p)}$ & $46.4 I_{p}$ & $S_{1}^{(p)}$ & $22.2 I_{p}$ \\
$S_{0}^{(d)}$ & $-460 I_{d}$ & $S_{1}^{(d)}$ & $384 I_{d}$ \\
\hline \hline
\end{tabular}

Using these pseudopotentials, all dipole moments we need are calculated, with which the necessary atomic parameters have been computed (see Appendixes A-C). The atomic parameters are listed in Tables III and IV for Rb and Cs. Under the influence of the strong dressing laser, the characteristic width of the states is predominantly determined by the ionization width of $|1\rangle, \Gamma_{1}^{(d)}\left(=\Gamma_{1 c_{1}}^{(d)}+\Gamma_{1 c_{2}}^{(d)}\right)$. From Tables III and IV, we find that the widths of $|1\rangle$ are $5 \mathrm{GHz}$ and $15 \mathrm{GHz}$ for $\mathrm{Rb}$ and $\mathrm{Cs}$, respectively, at $I_{d}=10^{9} \mathrm{~W} / \mathrm{cm}^{2}$. Obviously, these widths are broader than the hyperfine splittings of the ground state of $\mathrm{Rb}(3 \mathrm{GHz})$ and $\mathrm{Cs}(9 \mathrm{GHz})$. Therefore, at the intensity of $I_{d} \sim 10^{9} \mathrm{~W} / \mathrm{cm}^{2}$ and above, the hyperfine splittings can be safely neglected. At lower intensities, however, the hyperfine splittings have to be taken into account in a rigorous sense, but we did not elaborate it, since the primary objective of this paper is to study the role of spin-orbit interaction and the dressing laser with sufficient intensity in the production of spin-polarized photoelectrons.

\section{B. Photoelectron yield}

Now, with the atomic parameters calculated in Sec. III A, a set of time-dependent amplitude equations, Eqs. (6)-(8), is solved. Using the obtained solutions for $u_{0}(t), u_{1}(t)$, and $u_{2}(t)$, spin-up/-down photoelectron yields can be calculated from Eqs. (9)-(12). Before presenting the numerical results, we define the degree of spin polarization of photoelectrons as

$$
P=\frac{R_{c_{2}}+R_{c_{3}}+R_{c_{4}}-R_{c_{1}}}{R_{c_{2}}+R_{c_{3}}+R_{c_{4}}+R_{c_{1}}}
$$

TABLE IV. Atomic parameters for Cs $6 s-8 s$ system. $\Omega$ in $\mathrm{rad} / \mathrm{s}$, $\Gamma$ in $\mathrm{s}^{-1}, S$ in $\mathrm{rad} / \mathrm{s}$, and $I_{d}$ in $\mathrm{W} / \mathrm{cm}^{2}$.

\begin{tabular}{lccc}
\hline \hline$\Omega_{c_{1}}$ & $-0.81 \sqrt{I_{p} I_{d}}$ & $q_{c_{1}}$ & -4.07 \\
$\Omega_{c_{2}}$ & $3.48 \sqrt{I_{p} I_{d}}$ & $q_{c_{2}}$ & 7.67 \\
\hline$\Gamma_{0 c_{1}}^{(p)}$ & $0.586 I_{p}$ & $\Gamma_{1 c_{1}}^{(d)}$ & $0.273 I_{d}$ \\
$\Gamma_{0 c_{2}}^{(p)}$ & $0.0562 I_{p}$ & $\Gamma_{1 c_{2}}^{(d)}$ & $14.67 I_{d}$ \\
$\Gamma_{2 c_{4}}^{(p)}$ & $1.21 I_{p}$ & $\Gamma_{1 c_{3}}^{(p)}$ & $3.76 \times 10^{-3} I_{p}$ \\
$S_{0}^{(p)}$ & $35.8 I_{p}$ & $S_{1}^{(p)}$ & $25.4 I_{p}$ \\
$S_{0}^{(d)}$ & $-855 I_{d}$ & $S_{1}^{(d)}$ & $343 I_{d}$ \\
\hline \hline
\end{tabular}




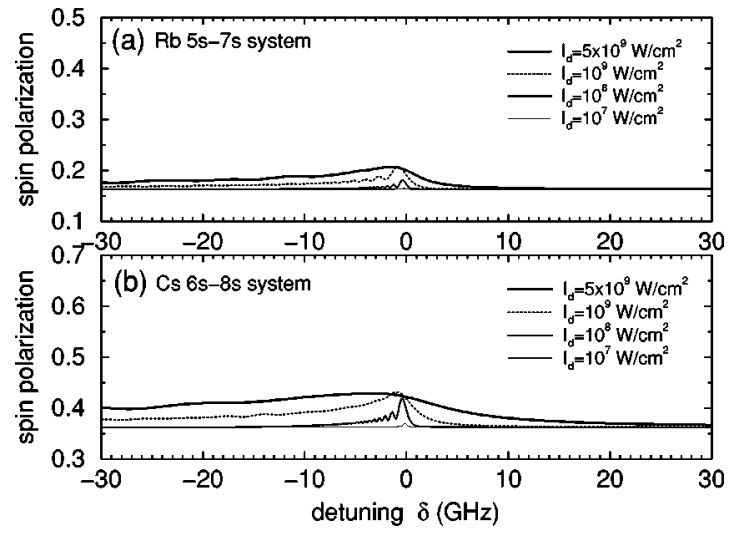

FIG. 3. Variation of spin polarization as a function of twophoton detuning at four different peak intensities for the dressing laser, $I_{d}=10^{7}$ (thin solid line), $I_{d}=10^{8}$ (solid line), $I_{d}$ $=10^{9} \mathrm{~W} / \mathrm{cm}^{2}$ (dotted line), and $I_{d}=5 \times 10^{9} \mathrm{~W} / \mathrm{cm}^{2}$ (thick solid line). Peak intensity of the probe laser is kept to be $I_{p}$ $=10^{7} \mathrm{~W} / \mathrm{cm}^{2}$. Pulse duration (Gaussian, FWHM) is $4 \mathrm{~ns}$ for both probe and dressing lasers. (a) Rb $5 s-7 s$ system and (b) Cs $6 s-8 s$ system.

where $R_{c_{2}}, R_{c_{3}}$, and $R_{c_{4}}$ are the spin-up photoelectron yields from $|0\rangle$ and $|1\rangle,|2\rangle$, and $|1\rangle$, respectively. $R_{c_{1}}$ is the spindown photoelectron yield from $|0\rangle$. Note that the effect of the dressing field is contained in the third terms of Eqs. (9) and (10).

Figure 3(a) shows the variation of spin polarization $P$ for the $\mathrm{Rb} 5 s-7 s$ system as a function of laser detuning $\delta(\mathrm{GHz})$ for four different peak intensities of the dressing laser, $I_{d}$ $=10^{7}$ (thin solid line), $10^{8}$ (solid line), and $10^{9}$ (dotted line), and $5 \times 10^{9} \mathrm{~W} / \mathrm{cm}^{2}$ (thick solid line). Peak intensity of the probe laser has been kept to be $I_{p}=10^{7} \mathrm{~W} / \mathrm{cm}^{2}$ for all curves. Pulse duration is taken to be 4 ns [Gaussian, full width at half maximum (FWHM)] for both lasers. Due to the intensity-dependent and naturally time-dependent ac Stark shifts, the net two-photon detuning cannot be maintained constant during the rise and fall of the pulse. Therefore, most of the laser-induced structures have been smeared out, and an enhancement of spin polarization turned out to be very small. Similar results are obtained for the Cs $6 s-8 s$ system [Fig. 3(b)]. In order to circumvent the problem of the ac Stark shifts which are mainly due to the intense dressing laser, we now employ a longer pulse for the dressing laser, $10 \mathrm{~ns}$, compared with the probe laser, 1 ns. Results are shown in Fig. 4(a) for $\mathrm{Rb}$. When the dressing laser intensity is small, $I_{d}=10^{7} \mathrm{~W} / \mathrm{cm}^{2}$, the spin polarization is practically constant, since the photoelectron yields $R_{c_{1}}$ and $R_{c_{2}}$ are dominated by the first terms of Eqs. (9) and (10). $\left(R_{c_{3}}\right.$ and $R_{c_{4}}$ do not depend on the dressing laser intensity, but contribute as a background.) As the dressing laser intensity increases, the third term as well as the second term in Eqs. (9) and (10) become non-negligible, and a significant structure shows up in spin polarization as a function of laser detuning $\delta$. Similar results are obtained for Cs. The main difference between $\mathrm{Rb}$ and $\mathrm{Cs}$ is that the spin-orbit interaction is larger for Cs and therefore, spin polarization at far-off resonance $(\delta$ $=10 \mathrm{GHz})$ is larger for $\mathrm{Cs}(37 \%)$ than $\mathrm{Rb}(17 \%)$. This is
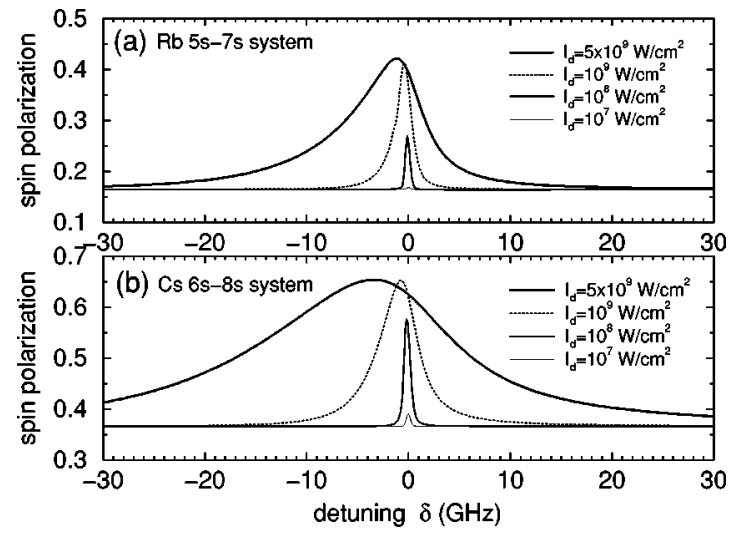

FIG. 4. Variation of spin polarization as a function of twophoton detuning at four different peak intensities for the dressing laser, $I_{d}=10^{7}$ (thin solid line), $I_{d}=10^{8}$ (solid line), $I_{d}$ $=10^{9} \mathrm{~W} / \mathrm{cm}^{2}$ (dotted line), and $I_{d}=5 \times 10^{9} \mathrm{~W} / \mathrm{cm}^{2}$ (thick solid line). Peak intensity of the probe laser is kept at $I_{p}=10^{7} \mathrm{~W} / \mathrm{cm}^{2}$. Pulse duration (Gaussian, FWHM) is $1 \mathrm{~ns}$ for the probe and $10 \mathrm{~ns}$ for the dressing lasers. (a) Rb $5 s-7 s$ system. (b) Cs $6 s-8 s$ system.

purely due to the spin-orbit interaction in the continuum without the effect of dressing laser. In addition, the laserinduced structure started to emerge at a lower dressing laser intensity for Cs, since the two-photon Rabi frequency $\Omega$ $\left(=\Omega_{c_{1}}+\Omega_{c_{2}}\right)$ for $\mathrm{Cs}$ is larger than Rb by more than a factor of 2. In terms of the enhancement factor of spin polarization caused by the dressing laser, it is 2.6 for $\mathrm{Rb}$ and 1.8 for Cs at $I_{p}=10^{7} \mathrm{~W} / \mathrm{cm}^{2}$ with $1 \mathrm{~ns}$ duration and $I_{d}=10^{9} \mathrm{~W} / \mathrm{cm}^{2}$ with $10 \mathrm{~ns}$ duration. In order to obtain a more physical understanding, photoelectron yields into each $R_{c_{1}}, R_{c_{2}}, R_{c_{3}}$, and $R_{c_{4}}$ are plotted in Fig. 5 as a function of detuning $\delta$. In each of Figs. 5(a) and 5(b), three curves correspond to $R_{c_{1}}$ (thin line), $R_{c_{2}}$ (solid line), and $R_{c_{3}}$ (dot-dashed line), respectively. ( $R_{c_{4}}$ is very small and practically zero in this scale.) It is perhaps interesting to point out that the laser-induced struc-

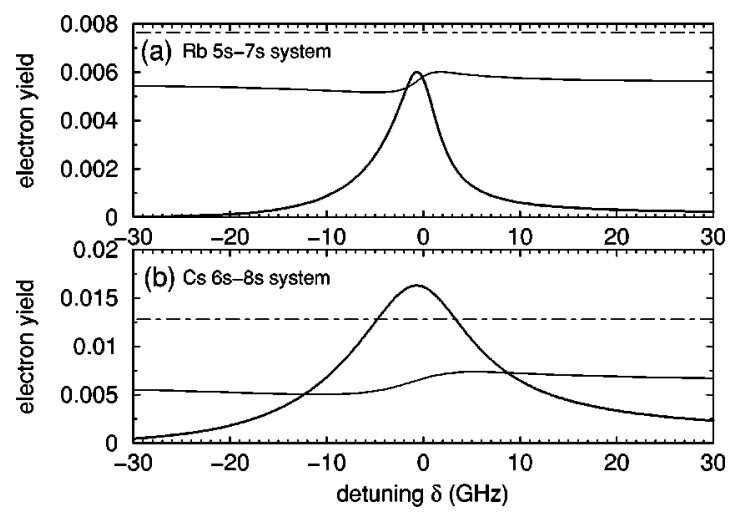

FIG. 5. Photoelectron yields into each $R_{c_{1}}$ (thin line), $R_{c_{2}}$ (solid line), $R_{c_{3}}$ (dot-dashed), and $R_{c_{4}}$ as a function of two-photon detuning $\delta$. $\left(R_{c_{4}}\right.$ is very small and practically zero in this scale.) $I_{p}$ $=10^{7} \mathrm{~W} / \mathrm{cm}^{2}$ and $I_{d}=5 \times 10^{9} \mathrm{~W} / \mathrm{cm}^{2}$. Pulse duration is $1 \mathrm{~ns}$ (FWHM, Gaussian) for the probe and $10 \mathrm{~ns}$ for the dressing lasers. (a) $\mathrm{Rb} 5 s-7 s$ system. (b) Cs $6 s-8 s$ system. 
ture of the photoelectron yield from $|0\rangle$ with up spin $R_{c_{2}}$ [solid lines in Figs. 5(a) and 5(b)], is much more significant compared with that of the photoelectron yield from $|0\rangle$ with down spin $R_{c_{1}}$ [thin lines in Figs. 5(a) and 5(b)], although both structures originate from the dressing laser [see Fig. 1(b)]. In terms of the perturbation theory this can be easily understood, with the help of Fig. 1(b), as follows: Starting from $|0\rangle$, the lowest-order ionization processes are $|0\rangle$ $\rightarrow\left|c_{1}\right\rangle$ and $|0\rangle \rightarrow\left|c_{2}\right\rangle$ for ionization into $\left|c_{1}\right\rangle$ and $\left|c_{2}\right\rangle$, respectively. The second-lowest-order ionization processes require three photons. For ionization into the continuum $\left|c_{1}\right\rangle$, it is represented by $|0\rangle \rightarrow\left|c_{1}\right\rangle \rightarrow|1\rangle \rightarrow\left|c_{1}\right\rangle$ and $|0\rangle \rightarrow\left|c_{2}\right\rangle$ $\rightarrow|1\rangle \rightarrow\left|c_{1}\right\rangle$, while ionization into the continuum $\left|c_{2}\right\rangle$ is represented by $|0\rangle \rightarrow\left|c_{2}\right\rangle \rightarrow|1\rangle \rightarrow\left|c_{2}\right\rangle$ and $|0\rangle \rightarrow\left|c_{1}\right\rangle \rightarrow|1\rangle$ $\rightarrow\left|c_{2}\right\rangle$. Obviously, the ionization into the continuum $\left|c_{2}\right\rangle$ is more sensitive to the dressing laser intensity, since the strong coupling between $|1\rangle$ and $\left|c_{2}\right\rangle$ by the dressing laser plays a more important role. Once we realize this, it is not surprising that the laser-induced structure into the spin-up continuum $\left|c_{2}\right\rangle$ is much more eminent, namely, more influenced by the dressing field, than that into the spin-down continuum $\left|c_{1}\right\rangle$. Mathematically, the above argument is even more clear from Eqs. (9) and (10), in which $\left|\Omega_{c_{1}}\right|<\left|\Omega_{c_{2}}\right|$ and also $\Gamma_{0 c_{1}}^{(p)}$ $\gg \Gamma_{0 c_{2}}^{(p)}$ : Assuming a weak probe field as it is now, contribution of the third term in Eqs. (10) relative to the first and the second terms is much larger than that of the third term in Eqs. (9) for sufficiently strong dressing laser intensity.

\section{SUMMARY}

In summary, we have studied the role of spin-orbit interaction in the production of spin-polarized photoelectrons under the action of a dressing laser. We have found that the spin-orbit interaction in the continuum is essential for the laser-induced structure to emerge. Without spin-orbit interaction, dressing of the excited state into the continuum does not occur, since the photoionization from the ground ${ }^{2} S_{1 / 2}\left(m_{j}\right.$
$=-1 / 2)$ state and an excited ${ }^{2} S_{1 / 2}\left(m_{j}=1 / 2\right)$ state by the circularly polarized probe and linearly polarized dressing lasers, respectively, go to different spin states, although both spin states belong to the same $p$ continuum with $m_{j}=1 / 2$. Specific numerical calculations have been carried out for the $\mathrm{Rb} 5 s-7 s$ and and Cs $6 s-8 s$ systems. In both cases, enhancement about a factor of 2 in the degree of spin polarization has been obtained by the introduction of a dressing laser at the intensity of $>10^{9} \mathrm{~W} / \mathrm{cm}^{2}$. Naturally, spin polarization of the Cs system turned out to be higher than that of the $\mathrm{Rb}$ system, since the spin-orbit interaction is larger for Cs. In order to avoid the undesired intensity-dependent ac Stark shifts, it is important that the pulse duration of the dressing laser is longer than that of the probe laser so that the twophoton detuning is maintained nearly constant during the photoelectron emission.

\section{ACKNOWLEDGMENT}

The work by T.N. was supported by a Grant-in-Aid for scientific research from the Ministry of Education and Science of Japan.

\section{APPENDIX A: IONIZATION WIDTHS}

Because of the spin-orbit interaction in the continuum, all the transition matrix elements become $j$ dependent. In the appendixes, we give explicit expressions for all matrix elements used in this work. In this appendix, we explicitly show that the bound-free matrix elements into the spin-up state (indicated by $\uparrow$ ) such as $\left\langle\vec{k} ; m_{j}=1 / 2, \uparrow\left|r_{+}\right| S_{1 / 2}, m_{j}=-1 / 2\right\rangle$ vanish in the limit of no spin-orbit interaction. Needless to say, no spin-orbit interaction implies that all matrix elements are $j$ independent, i.e., $R_{s \rightarrow k p_{1 / 2}} \simeq R_{s \rightarrow k p_{3 / 2}}$. This means that the photoelectron from a spin-down bound state is necessarily spin down in the absence of spin-orbit interaction, as we have mentioned in the Introduction. Ionization widths (in units of $s^{-1}$ ) are calculated from the relations,

$$
\begin{aligned}
& \Gamma_{0 c_{1}}^{(p)}=0.589 I_{p}\left(2 \pi\left|\int \Omega_{\text {solid }}\left\langle\vec{k} ; m_{j}=1 / 2, \downarrow\left|r_{+}\right| n_{0} S_{1 / 2}, m_{j}=-1 / 2\right\rangle\right|^{2}\right)=0.589 I_{p}\left(2 \pi\left|\frac{2}{3 \sqrt{3}}\left(2 R_{n_{0} s \rightarrow k p_{1 / 2}}+R_{n_{0} s \rightarrow k p_{3 / 2}}\right)\right|^{2}\right), \\
& \Gamma_{0 c_{2}}^{(p)}=0.589 I_{p}\left(2 \pi\left|\int \Omega_{\text {solid }}\left\langle\vec{k} ; m_{j}=1 / 2, \uparrow\left|r_{+}\right| n_{0} S_{1 / 2}, m_{j}=-1 / 2\right\rangle\right|^{2}\right)=0.589 I_{p}\left(2 \pi\left|\frac{2}{3 \sqrt{6}}\left(-R_{n_{0} s \rightarrow k p_{1 / 2}}+R_{n_{0} s \rightarrow k p_{3 / 2}}\right)\right|^{2}\right) \\
& \Gamma_{1 c_{1}}^{(d)}=0.589 I_{d}\left(2 \pi\left|\int \Omega_{\text {solid }}\left\langle\vec{k} ; m_{j}=1 / 2, \downarrow\left|r_{0}\right| n_{1} S_{1 / 2}, m_{j}=1 / 2\right\rangle\right|^{2}\right)=0.589 I_{d}\left(2 \pi\left|\frac{2}{3 \sqrt{6}}\left(-R_{n_{1} s \rightarrow k p_{1 / 2}}+R_{n_{1} s \rightarrow k p_{3 / 2}}\right)\right|^{2}\right) \\
& \Gamma_{1 c_{2}}^{(d)}=0.589 I_{d}\left(2 \pi\left|\int \Omega_{\text {solid }}\left\langle\vec{k} ; m_{j}=1 / 2, \uparrow\left|r_{0}\right| n_{1} S_{1 / 2}, m_{j}=1 / 2\right\rangle\right|^{2}\right)=0.589 I_{d}\left(2 \pi\left|\frac{2}{3 \sqrt{3}}\left(R_{n_{1} s \rightarrow k p_{1 / 2}}+2 R_{n_{1} s \rightarrow k p_{3 / 2}}\right)\right|^{2}\right)
\end{aligned}
$$




$$
\Gamma_{2 c_{3}}^{(p)}=0.589 I_{p}\left(2 \pi\left|\int \Omega_{\text {solid }}\left\langle\vec{k} ; m_{j}=1 / 2, \uparrow\left|r_{+}\right| n_{2} S_{1 / 2}, m_{j}=1 / 2\right\rangle\right|^{2}\right)=0.589 I_{p}\left(2 \pi\left|\frac{1}{\sqrt{3}} R_{n_{2} s \rightarrow k p_{3 / 2}}\right|^{2}\right)
$$

where $\Omega_{\text {solid }}$ is a solid angle of the outgoing photoelectron and $R_{n_{0} s \rightarrow k p_{1 / 2}}$ is the bound-free radial matrix element (in atomic units) between states $\left|n_{0}^{2} S_{1 / 2}, m_{j}=-1 / 2\right\rangle(=|0\rangle)$ and $\left|\vec{k} ; m_{j}=1 / 2, \downarrow\right\rangle$, etc. $I_{p}$ and $I_{d}$ are in $\mathrm{W} / \mathrm{cm}^{2} . r_{0}$ and $r_{+}$are the spherical components of the electric dipole moment of the valence electron, corresponding to the excitation by linearly polarized and right-circularly polarized radiations, respectively.

\section{APPENDIX B: TWO-PHOTON RABI FREQUENCIES}

In this appendix, we explicitly show that the two-photon Rabi frequency $\Omega\left(=\Omega_{c_{1}}+\Omega_{c_{2}}\right)$ between states $|0\rangle$ and $|1\rangle$ vanishes in the limit of no spin-orbit interaction, i.e., $R_{s \rightarrow k p_{1 / 2}} \simeq R_{s \rightarrow k p_{3 / 2}}$. This means that the dressing laser cannot induce a LICS, as we explained in the Introduction. The partial two-photon Rabi frequencies (in units of $\mathrm{rad} / \mathrm{s}$ ) via $\left|c_{1}\right\rangle$ and $\left|c_{2}\right\rangle$ are defined as

$$
\Omega_{c_{1}}=0.589 \sqrt{I_{p} I_{d}} \frac{\sqrt{2}}{27}\left(-2 R_{n_{0} s \rightarrow p_{1 / 2} \rightarrow n_{1} s}+R_{n_{0} s \rightarrow p_{3 / 2} \rightarrow n_{1} s}\right)
$$

$$
\Omega_{c_{2}}=0.589 \sqrt{I_{p} I_{d}} \frac{\sqrt{2}}{27}\left(-R_{n_{0} s \rightarrow p_{1 / 2} \rightarrow n_{1} s}+2 R_{n_{0} s \rightarrow p_{3 / 2} \rightarrow n_{1} s}\right)
$$

where $R_{n_{0} s \rightarrow p_{1 / 2} \rightarrow n_{1} s}$ and $R_{n_{0} s \rightarrow p_{1 / 2} \rightarrow n_{1} s}$ are the two-photon radial matrix elements between $\left|n_{0}^{2} S_{1 / 2}, m_{j}=-1 / 2\right\rangle(=|0\rangle)$ and $\left|n_{1}^{2} S_{1 / 2}, m_{j}=1 / 2\right\rangle(=|1\rangle)$ via intermediate states $p_{1 / 2}$ and $p_{3 / 2}$. The asymmetry parameters are obtained from the following relation,

$$
q_{c}=\frac{2 \Omega_{c}}{\sqrt{\Gamma_{0 c}^{(p)} \Gamma_{1 c}^{(d)}}}
$$

where $c=c_{1}$ or $c_{2}$.

\section{APPENDIX C: AC STARK SHIFTS}

The ac Stark shifts (in units of $\mathrm{rad} / \mathrm{s}$ ) are calculated from the relations

$$
\begin{gathered}
S_{0}^{(p)}=0.589 I_{p}\left(\sum_{\beta=n p_{1 / 2}, n p_{3 / 2}}\left|\frac{\left\langle\beta, m_{j}=1 / 2\left|r_{+}\right| n_{0} S_{1 / 2}, m_{j}=-1 / 2\right\rangle}{\omega_{0}+\omega_{p}-\omega_{\beta}}\right|^{2}\right)=0.589 I_{p}\left[\frac{2}{9}\left|R_{n_{0} s \rightarrow n p_{1 / 2}}\right|^{2}+\frac{1}{9}\left|R_{n_{0} s \rightarrow n p_{3 / 2}}\right|^{2}\right], \\
S_{0}^{(d)}=0.589 I_{d}\left(\sum_{\beta=n p_{1 / 2}, n p_{3 / 2}}\left|\frac{\left\langle\beta, m_{j}=-1 / 2\left|r_{0}\right| n_{0} S_{1 / 2}, m_{j}=-1 / 2\right\rangle}{\omega_{0}+\omega_{d}-\omega_{\beta}}\right|^{2}\right)=0.589 I_{d}\left[\frac{1}{9}\left|R_{n_{0} s \rightarrow n p_{1 / 2}}\right| 2+\frac{2}{9} \mid R_{\left.\left.n_{0} s \rightarrow n p_{3 / 2}\right|^{2}\right],}\right. \\
S_{1}^{(p)}=0.589 I_{p}\left(\sum_{\beta=n p_{3 / 2}}\left|\frac{\left\langle\beta, m_{j}=3 / 2\left|r_{+}\right| n_{1} S_{1 / 2}, m_{j}=1 / 2\right\rangle}{\omega_{1}+\omega_{d}-\omega_{\beta}}\right|^{2}\right)=0.589 I_{p}\left(\frac{1}{3}\left|R_{n_{1} s \rightarrow n p_{3 / 2}}\right|^{2}\right), \\
S_{1}^{(d)}=0.589 I_{d}\left(\sum_{\beta=n p_{1 / 2}, n p_{3 / 2}}\left|\frac{\left\langle\beta, m_{j}=1 / 2\left|r_{0}\right| n_{1} S_{1 / 2}, m_{j}=1 / 2\right\rangle}{\omega_{1}+\omega_{d}-\omega_{\beta}}\right|^{2}\right)=0.589 I_{d}\left[\frac{1}{9}\left|R_{n_{1} s \rightarrow n p_{1 / 2}}\right| 2+\frac{2}{9}\left|R_{n_{1} s \rightarrow n p_{3 / 2}}\right|^{2}\right] .
\end{gathered}
$$

[1] T. Nakajima and P. Lambropoulos, Europhys. Lett. 57, 25 (2002).

[2] T. Nakajima and N. Yonekura, J. Chem. Phys. 117, 2112 (2002).

[3] T. Nakajima, N. Yonekura, T. Kobayashi, Y. Matsuo, and Y. Fukuyama, Appl. Phys. Lett. (to be published).

[4] Yu.I. Heller and A.K. Popov, Opt. Commun. 18, 449 (1976).

[5] Bo-nian Dai and P. Lambropoulos, Phys. Rev. A 36, 5205 (1987); 39, 3704 (1989).
[6] P.L. Knight, M.A. Lauder, and B.J. Dalton, Phys. Rep. 190, 1 (1991).

[7] Y.L. Shao, D. Charalambidis, C. Fotakis, Jian Zhang, and P. Lambropoulos, Phys. Rev. Lett. 67, 3669 (1991).

[8] S. Cavalieri, F.S. Pavone, and M. Matera, Phys. Rev. Lett. 67, 3673 (1991).

[9] L.P. Yatsenko, T. Halfmann, B.W. Shore, and K. Bergmann, Phys. Rev. A 59, 2926 (1999).

[10] K. Böhmer, T. Halfmann, L.P. Yatsenko, D. Charalambidis, A. 
Horsmans, and K. Bergmann, Phys. Rev. A 66, 013406 (2001). [11] A.I. Andryushin and M.V. Fedorov, Zh. Eksp. Teor. Fiz. 75, 2037 (1978) [Sov. Phys. JETP 48, 228 (1978)].

[12] G. Alber, and P. Zoller, Phys. Rev. A 27, 1713 (1983).

[13] P. Lambropoulos and P. Zoller, Phys. Rev. A 24, 379 (1981).
[14] T. Nakajima, Jian Zhang, and P. Lambropoulos, J. Phys. B 30, 1077 (1997).

[15] W.R. Johnson, D.S. Guo, M. Idress, and J. Sapirstein, Phys. Rev. A 32, 2093 (1985). 